\title{
Über Pepsin-Glutinpepton.
}

Von

W. Scheermesser.

(Aus der chem. Abteilung des physiologischen Institutes der Universität Leipzig.) (Der Redaktion zugegangen am 14. Januar 1904.)

Auf Veranlassung des Herrn Prof. Dr. Siegfried habe ich mit Hilfe der Eisenmethode aus dem Leim durch peptische Verdauung ein neues Pepton dargestellt. ${ }^{1}$ ) Wenn auch die Darstellung zeitraubend und schwierig ist, so führt sie doch, nach Einarbeitung in die Methode, zu sicheren Resultaten.

\section{Verdauung der Gelatine.}

a) Ausführung.

Als Ausgangsmaterial zur Darstellung des Peptons aus Glutin verwendete ich beste französische Gelatine.

So wurde beispielsweise $1 \frac{1 / 2 \mathrm{~kg}}{}$ hiervon während der Nacht in kaltem Wasser eingeweicht und dann das Wasser gewechselt. Zuletzt wurde die Gelatine nochmals mit destilliertem Wasser ausgewaschen. Ich halte diese vorhergehende Behandlung deshalb für unbedingt notwendig, weil bei der Fabrikation der Gelatine unter Umständen Stoffe als Zucker, Glycerin u. a. hinzugefügt werden; Glycerin z. B. deshalb, um das Produkt geschmeidiger zu machen.

Die auf diese Weise gereinigte Gelatine wurde in Wasser von 70 bis $80^{\circ}$ gelöst, die Lösung durch ein Tuch geseiht und das Gesamtgewicht auf $15 \mathrm{~kg}$ gebracht. Dieser Flüssigkeit wurde soviel Salzsäure hinzugefügt, daß der Gehalt daran $0,4 \%$ betrug. Zwecks Sterilerhaltung der Gelatineflüssig-

1) Vgl. Diese Zeitschrift, Bd. XXXVII, S. 363 . 
keit wurden genügende Mengen Chloroform sowie alkoholische Thymollösung hinzugegeben. An jedem fünften Tage wurden $5 \mathrm{~g}$ «Pepsin puriss. Dr. G. Grübler» hinzugefügt und unter häufigem Umschütteln im Verdauungsbade bei $40^{\circ}$ verdaut. Im ganzen wurden $45 \mathrm{~g}$ Pepsin verwandt.

Schon am dritten Tage war äußerlich eine Veränderung der Gelatinelösung wahrnehmbar. War dieselbe bis dahin trübe und farblos, so wurde sie nach dieser Zeit völlig klar; ein geringer flockiger Niederschlag hatte sich zu Boden gesetzt; die Farbe der Lösung war gelblich.

b) Kontrolle der Verdaung.

Um zu sehen, wie bei diesem Versuch die Verdauung fortschritt, bezw. wie die Peptonbildung zunahm, entnahm ich von fünf zu fünf Tagen $100 \mathrm{ccm}$ von der Verdauungsflüssigkeit und salzte dieselbe mit Ammonsulfat in neutraler und in saurer Reaktion aus; von dieser Flüssigkeit ließ ich zu $50 \mathrm{ccm}$ aus einer Bürette eine Lösung hinzufließen, welche bestand aus

$50 \mathrm{~g}$ Eisenammoniakalaun,

200 . Ammonsulfat,

250 . Wasser.

Der sich bildende Niederschlag setzte sich sehr schnell $a b$, sodaß man das Ende der Titration leicht erkennen konnte. Später vervollkommnete ich die Methode noch dadurch, daß ich aus der zu titrierenden Flüssigkeit mit einem Glasstab einen Tropfen herausnahm und ihn in eine, Rhodankali enthaltende Schale brachte. Das Eisen wird von dem Pepton so energisch (in ammonsulfatgesättigter Lösung) in Beschlag genommen, daß der geringste Überschuß genügt, um die Rhodanlösung zu röten.

Ich gebe $\mathrm{zu}$, daß die Methode deshalb nicht ganz genau sein kann, weil in den Niederschlag noch kleine Mengen Albumosen mit hineingehen, immerhin läßt sich mit ihrer Hilfe das Fortschreiten der Verdauung recht gut kontrollieren. $\mathrm{Ob}$ sich die Methode auch für andere Eiweißkörper wird verwenden lassen, müßte noch erst nachgeprüft werden. Für die Glutinverdauung geht die Methode glatt und ergab folgende Zahlen: 


\begin{tabular}{|c|c|c|c|}
\hline $\mathrm{Nr}$. & Datum & Reaktion & $\begin{array}{c}\text { Verbrauchte } \\
\text { ccm }\end{array}$ \\
\hline 1 & 21. 4. 02 & \multirow{9}{*}{ 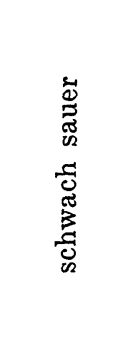 } & 0 \\
\hline 2 & 22. 4. 02 & & 0 \\
\hline 3 & 23. 4. 02 & & 1,1 \\
\hline 4 & 28. 4. 02 & & 1,2 \\
\hline 5 & 3. 5.02 & & 2,5 \\
\hline 6 & 7. 5.02 & & 2,7 \\
\hline 7 & 12. 5. 02 & & 3,0 \\
\hline 8 & 14. 5. 02 & & 3,0 \\
\hline 9 & 16. 5. 02 & & 2,8 \\
\hline
\end{tabular}

Aus den gefundenen Werten sieht man, daß Pepton nicht sofort durch Verdauung von Glutin gebildet wird - ich erhielt bei diesem Versuche nach den ersten zwei Tagen nicht den geringsten Niederschlag - vielmehr wird die bereits früher bestehende Anschauung bewiesen, daß erst aus den Albumosen Pepton entsteht. Nach ca. 20 Tagen wurde die Peptonmenge scheinbar nicht weiter vermehrt.

Über den Verbrauch an Salzsäure während der Verdauung gab folgender Versuch Aufschluß. Es wurde 11 einer 5\% igen Gelatinelösung hergestellt, welche $0,4 \% \mathrm{HCl}$ enthielt. Zwecks Sterilerhaltung wurde ca. $1 \mathrm{ccm}$ Chloroform, in welchem etwas Thymol gelöst war, hinzugegeben und umgeschüttelt. Diese Lösung wurde in zwei Teile geteilt und in zwei Glasstöpselflaschen in einen elektrisch heizbaren Thermostaten gestellt, dessen Temperatur vermittelst eines von mir konstruierten, der Firma F. Hugershoff in Leipzig durch D.R.G.M. geschützten Elektrothermoregulators genau auf $40^{\circ}$ eingestellt wurde.

In das eine Glas brachte ich, nachdem die Temperatur bei $40^{\circ}$ konstant war, $5 \mathrm{~g}$ "Pepsin puriss. Dr. Grübler $1: 7000$ », während in das andere zur Kontrolle dienende Glas kein Pepsin kam. An jedem dritten Tage prüfte ich mit einem sehr schwach gefärbten «Nissl»-Kongopapier (Dr. Grüblers No. I), ob die Salzsäure gebunden war; regelmäßig nach drei Tagen trat keine Bläuung des Kongopapiers ein, während Lackmus stark gerötet wurde. Ich ließ dann aus einer Bürette jedesmal so lange norm-HCl hinzufließen, bis ein deutlicher Farbenumschlag des Kongofarbstoffes von Rot in Blau eintrat. Der Verbrauch an 
Salzsäure war in dem gleichen Zeitraum im Anfang ganz bedeutend größer, als am Ende. Es betrug die verbrauchte norm-HCl

$$
\begin{aligned}
& \text { nach } 3 \text { Tagen }=12,7 \mathrm{ccm} \\
& \text { \ } 6=12,3 \text { » } \\
& \triangleright 9 \triangleright=8,5 \text { ॰ } \\
& \text { \12 =7,4 》 } \\
& \gg 15 \gg=5,8 \text { 》 } \\
& \text { 》 } 18 \text { > }=5,6 \text { 》 } \\
& \text { \ } 21 \text { 》 }=5,5 \text { 》 } \\
& \text { » } 24 \text { > }=4,7 \text { » }
\end{aligned}
$$

nach 27 Tagen $=4,1 \mathrm{ccm}$

$$
\begin{aligned}
& \text { » } 30 \text { » }=3,5 \text {, } \\
& \text { \33 }>=3,0 \text {, } \\
& \triangleright 36 \text { » }=2,0 \text { » } \\
& \triangleright 39 \text { > }=2,2 \text {. } \\
& \triangleright 42>=0,0 \text { > } \\
& \text { \45 》 }=0,0 \text {, }
\end{aligned}
$$

Trage ich diese Zahlen in ein Koordinatensystem ein, so ergibt sich für den $\mathrm{HCl}-\mathrm{Verb}$ rauch bei der Pepsinverdauung von Glutin eine anfangs rapid, später weniger schnell abfallende Kurve. Addiere ich den gesamten Verbrauch an $\mathrm{HCl}$, so resultiert aus vorliegendem Versuch, daß zur Verdauung von $25 \mathrm{~g}$ Glutin mit $5 \mathrm{~g}$ Pepsin in 5\% \% iger wässeriger Lösung bei $+40^{\circ}$ insgesamt 4,82 $\mathrm{g} \mathrm{HCl}$ benötigt wurden.

Nach ca. 5 Wochen wird keine Salzsäure mehr verbraucht.

5 Tage nach Abschluß des Versuchs setzte ich zu der Verdauungsflüssigkeit nochmals $2 \mathrm{~g}$ Pepsin hinzu, worauf nach 20 Stunden nochmals $1,3 \mathrm{ccm}$ norm-HCl nötig waren, um Bläuung des Kongopapiers zu erreichen; seitdem verschwand die HCl-Reaktion auch innerhalb einer Woche nicht mehr.

Salzsäureverbrauch während der Verdauung von Glutin.

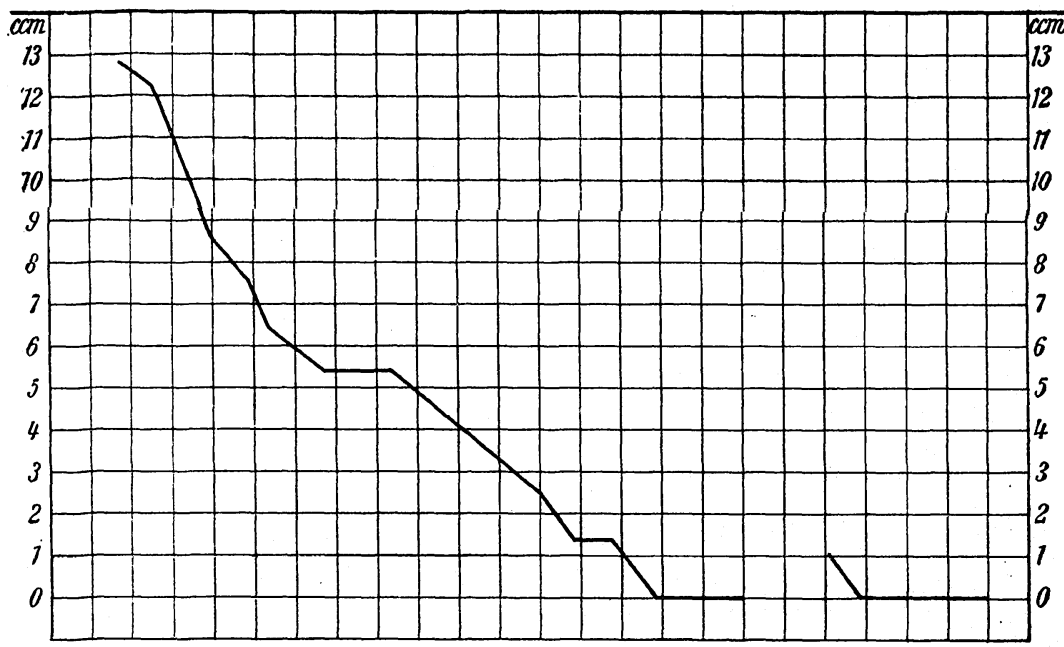


Das Drehvermögen nahm nicht wesentlich während der Verdauung ab. Die Gelatinelösung drehte vor der Verdauung am Saccharimeter von Schmidt \& Haens ch bei Na-Licht im $10 \mathrm{~cm}-$ Rohr anfangs $7,3^{\circ}$ (Rohrzucker), nachher, zum Schluß 6,9 ${ }^{\circ}$.

\section{Isolierung des Pepsin-Glutinpeptons.}

Nach Beendigung der Verdauung neutralisierte ich die Flüssigkeit mit Ammoniak und filtrierte sie. Durch festes Ammonsulfat wurde bei $40^{\circ}$ die größte Menge Albumosen ausgesalzen. Diese schieden sich in Form eines zähen, klebrigen Schleims $a b$, der zum größten Teil auf der Oberfläche sich ansammelte und in der Hauptsache mechanisch entfernt wurde. Die Flüssigkeit wurde filtriert und war danach völlig farblos und klar; sie wurde so lange mit (mit Ammonsulfat gesättigtem) Ammoniak versetzt, bis eine Trübung nicht mehr entstand. Nach 24 Stunden wurde filtriert. $\mathrm{Zu}$ dieser Lösung wurde konzentrierte Schwefelsäure (verdünnt mit gleichen Teilen Ammonsulfatlösung) bis zur neutralen Reaktion gegeben, nach 24 Stunden wurde filtriert.

Diese Flüssigkeit, welche nun fast albumosefrei war, wurde mittels Ammoniak bis zur schwach sauren Reaktion abgestumpft. Durch Zusatz von feinpulverisiertem Eisenammoniakalaun wurde, unter kräftigem Umrühren vermittelst eines mechanischen Rührwerkes, hierin ein Niederschlag erzeugt. Der Zusatz von pulverisiertem Eisenammoniakalaun wurde so lange fortgesetzt, bis in einer herausgenommenen Probe keine Trübung bezw. Fällung mehr stattfand. Nach Beendigung dieses Einrührens hatte die Lösung eine weingelbe Farbe. Der Eisenniederschlag unterscheidet sich von den Eisenniederschlägen der Peptone aus Casein sowohl als auch aus Fibrin; er fiel anfangs flockig, wurde dann körnig, war dunkelbraun bis rot und setzte sich nach wenigen Augenblicken schon zu Boden. Er wurde abgenutscht und so lange mit gesättigter Ammonsulfatlösung gedeckt, bis das Filtrat völlig chlorfrei war.

Der, soweit als möglich abgenutschte, körnige Niederschlag entsprach einem Verbrauch von $79 \mathrm{~g}$ Eisenammoniakalaun und wog $455 \mathrm{~g}$. Er wurde in einer Reibschale mit Wasser zu einem Brei angerührt und dann unter Umrühren in 21 Wasser gelöst. 
Nach M. Siegfried ${ }^{1}$ ) lassen sich die letzten Albumosenreste so nicht völlig entfernen, dagegen werden sie vollständig ausgeschieden, wenn der erhaltene Eisenniederschlag mit Ammoniak zersetzt, das Filtrat von $\mathrm{Fe}(\mathrm{OH})_{3}$ mit Ammonsulfat gesättigt und einmal Ammoniak, dann konzentrierte Schwefelsäure hinzugefügt wird.

Demgemäß zersetzte ich den in 21 Wasser gelösten Peptoneisenniederschlag mit Ammoniak unter Umrühren bei $40^{\circ}$. Hierbei schied sich braunes Eisenhydroxyd $a b$, welches nach längerem Stehen abgenutscht wurde. Dasselbe wurde mit Wasser zu einem dünnen Brei angerieben und auf dem Wasserbade auf ca. $50^{\circ}$ erwärmt. Danach wurde abgenutscht, ausgewaschen und die Waschwässer mit dem Filtrate vom Eisenhydroxyd vereinigt. Die Flüssigkeit wurde wiederum sowohl in alkalischer als auch neutraler, als auch $\mathrm{H}_{2} \mathrm{SO}_{4}$-saurer Lösung mit Ammonsulfat ausgesalzen. Da bei jeder der drei Operationen die Abscheidung der Restalbumosen nur in Form undurchsichtiger Wolken stattfand, so war die Filtration, auch nach längerem Stehen der Lösung, mit großen Schwierigkeiten verknüpft: das Filter belegte sich bald mit einer schleimigen Schicht, die den Hindurchgang der Flüssigkeit völlig verhinderte. Bei dem Wechsel des Filters war dagegen ein größerer Verlust unvermeidlich.

Ich half mir daher folgendermaßen: Ich kochte zerkleinerte Filtrierpapierschnitzel mit Ammonsulfatlösung längere Zeit und verwandelte dieselben dadurch in einen dünnen Brei. Diesen setzte ich der zu filtrierenden Flüssigkeit zu und rührte dieselbe mit Hilfe eines Elektromotors mit Rührwerk schnell und kräftig. Die Anwendung dieses Kunstgriffes ermöglichte es, daß nach ca. 10 Minuten die Lösung völlig klar war; die Albumosenpartikelchen hatten sich zusammengeballt und bildeten mit dem Filtrierpapierbrei eine zusammenhängende, am Rührer festhaftende Masse.

In diese nun völlig albumosefreie Peptonlösung wurde bei saurer Reaktion wiederum gepulverter Eisenammoniakalaun unter Umrühren eingetragen und, wie vorher, ein Eisenniederschlag gebildet. Diesmal betrug der Verbrauch an Eisen-

1) M. Siegfried, l. c. 
ammoniakalaun $73 \mathrm{~g}$, während der, so weit als möglich abgesaugte mit konzentrierter Ammonsulfatlösung gewaschene Niederschlag $442 \mathrm{~g}$ wog. Die Farbe war etwas dunkler als die des nicht umgefällten Eisenniederschlages. Dieser wurde gelöst, das Eisenhydroxyd durch Ammoniak abgeschieden. Das Ammonsulfat wurde in Wasser mit Barythydrat zersetzt, das $\mathrm{BaSO}_{4}$ abfiltriert, der Überschuß an $\mathrm{Ba}(\mathrm{OH})_{2}$ durch Einleiten von $\mathrm{CO}_{2}$ als $\mathrm{BaCO}_{3}$, das abfiltriert wurde, entfernt. Die Flüssigkeit, ca. 7 l, enthielt jetzt außer dem Pepton nur noch Ammonkarbonat. Sie wurde im Vacuum bei $27-33^{0}$ eingedampft.

Der Rückstand wurde mit wenig warmem Wasser aufgenommen und filtriert. Der dünne Sirup wurde dann mit 99\% igem Alkohol in solchem Verhältnis gemischt, daß das Verhältnis an

$$
\begin{gathered}
\text { Pepton : Wasser : Alkohol }= \\
10: 15: 5
\end{gathered}
$$

war. Nach Hinzufügen von $1 \mathrm{ccm}$ Eisessig wurde die Mischung unter Umrühren in 41 Alkohol $(99 \%)$ langsam eingegossen: es schied sich das Glutinpepton in weißen Flocken aus. Nachdem sich dasselbe, nach 24 Stunden etwa, zu Boden gesetzt hatte, wurde es abgesaugt, erst mit genügend Alkohol, dann mit Äther ausgewaschen und im Exsiccator einige Wochen getrocknet. Die Ausbeute betrug in diesem Fall $39 \mathrm{~g}$.

Die hier als Beispiel angeführte Darstellung war die dritte Darstellung.

\section{Eigenschaften des Pepsin-Glutinpeptons.}

Das erhaltene Pepton stellte ein schneeweißes Pulver dar, welches einen angenehmen, sauren Geschmack besitzt, völlig geruchlos ist und blaues Lackmuspapier stark rötet. Es ist in Wasser klar löslich und zwar in jedem Verhältnis, obgleich es sich nicht $\mathrm{zu}$ schnell darin auflöst. In absolutem Alkohol ist es nur in Spuren löslich.

Vollständig unlöslich ist es in wasserfreiem Äther, Benzol, Chloroform.

In Methylalkohol ist es etwas löslich, ebenso in Weingeist, und zwar umsomehr, je wasserhaltiger die letztgenannten Lösungsmittel sind. 
In gesättigter Ammoniumsulfatlösung ist das Pepton ebenso schnell als in Wasser löslich; erst bei stärkster Konzentration, z. B. 1 Teil Pepton und 2 Teile Wasser oder auch Ammoniumsulfatlösung, ist die Lösung schwach gelblich gefärbt, andernfalls ist sie farblos. In gesättigter Ammonsulfatlösung bewirkt weder der Zusatz von Ammoniak noch von konzentrierter Schwefelsäure eine Fällung, nicht einmal eine Trübung - ein Zeichen, daß das Präparat völlig albumosefrei war.

Die Reaktionen des Peptons sind folgende:

Biuretreaktion: stark positiv, auch beim Kochen.

Molischsche Reaktion: kaum vorhanden. Zusatz von Ferrocyankalilösung und Essigsäure bewirkte keine Trübung. Mit Gerbsäure gab die Peptonlösung eine starke Fällung, der Niederschlag war in Essigsäure löslich. Auf Zusatz einer geringen Menge Pikrinsäure entstand kein Niederschlag, auf Zusatz größerer Mengen Pikrinsäure eine Trübung, aber keine Fällung. Beim Erwärmen verschwand die Trübung. Merkurichlorid negativ, ebenso Bleiessig und Silbernitrat. In verdünnten Peptonlösungen fällte Phosphorwolframsäure nicht, in konzentrierten Lösungen dagegen stark. Völlig negativ waren die Reaktionen mit Metaphosphorsäure, die Xanthoproteinreaktion und die Adamkiewiczreaktionen.

Nachstehend sei eine Übersicht der Ausbeuten des Peptons bei den verschiedenen Darstellungen angegeben.

\section{Darstellung.}

Verdaut wurden bei $37,5^{\circ}$ während 12 Tagen bei $0,4 \% \mathrm{HCl}: 500 \mathrm{~g}$ Gelatine in $4500 \mathrm{~g}$ Wasser mit $10 \mathrm{~g}$ Pepsin Dr. Grübler.

Ausbeate: $1,3 \mathrm{~g}$ Pepton.

II. Darstellung.

Verdaut wurden bei $40^{\circ}$ während 27 Tagen bei $0,4 \% \mathrm{HGl}: 500 \mathrm{~g}$ Gelatine in $3000 \mathrm{~g}$ Wasser mit $15 \mathrm{~g}$ Pepsin Dr. Grübler.

\section{Ausbeute: $13 \mathrm{~g}$ Pepton.}

Da dieses Pepton nicht völlig albumosenfrei war, wurde es nochmals ausgesalzen. Durch die bei diesen Manipulationen unvermeidlichen Verluste verringerte sich die Ausbeute an Pepton. Sie betrug nach dem Trocknen $2,8 \mathrm{~g}$. 


\section{Darstellung.}

Verdaut wurden bei $40^{\circ}$ während 27 Tagen bei $0,3 \% \mathrm{HCl}: 1^{1 / 2} \mathrm{~kg}$ Gelatine in $15 \mathrm{l}$ Wasser mit $45 \mathrm{~g}$ Pepsin Dr. Grübler (1:7000).

Ausbeute: $39 \mathrm{~g}$ Pepton.

\section{Darstellung.}

Verdaut wurden bei $40^{\circ}$ während 30 Tagen bei $0,4 \% \mathrm{HCl}: 1 \mathrm{~kg}$ Gelatine in $15 \mathrm{l}$ Wasser mit $45 \mathrm{~g}$ Pepsin Dr. Grübler (1: 7000).

Ausbeute: 19,2 g Pepton.

\section{Darstellung.}

Verdaut wurden bei $40^{\circ}$ während 30 Tagen bei $0,20,0 \mathrm{HCl}: 500 \mathrm{~g}$ Gelatine in 201 Wasser.

Statt des Pepsins verwendete ich die Schleimhaut von vier Schweinemagen.

Die Magen wurden, nachdem sie umgestülpt waren, mit kaltem Wasser kurze Zeit gewaschen und dann vermittelst eines scharfkantigen Uhrglases ausgekrat/zt. Der Schleim wurde in ein Pulverglas gebracht, mit Wasser vermischt und so viel Salzsäure hinzugefügt, daß die Flüssigkeit $0,2 \%$ davon enthielt. Nach Zusatz von Chloroform und Thymol blieb das gut verschlossene Glas 12 Stunden im Brutschrank bei $40^{\circ}$ stehen.

Nach dieser Zeit wurde die Flüssigkeit filtriert und vermittelst einer Fibrinflocke auf ihre Verdauungsfähigkeit geprüft. Da das Fibrin leicht verdaut wurde, wurde mit dieser Flüssigkeit die Gelatinelösung versetzt und dann noch soviel $\mathrm{HCl} \mathrm{zu-}$ gefügt, daß die Gesamtflüssigkeit $0,2 \%$ davon enthielt.

\section{Ausbeute: $5,8 \mathrm{~g}$ Pepton.}

Außerdem verarbeitete ich noch ein mir von Herrn Prof. Siegfried gütigst zur Verfügung gestelltes Rohpepton, welches, da es nicht albumosefrei war, ausgesalzen und umgefällt wurde. Es sei mit IV bezeichnet.

Die geringen Ausbeuten erklären sich einmal durch die große Widerstandsfähigkeit des Leims gegen Pepsin, sodann aus dem Umstande, daß die Methode der Darstellung keine quantitative ist.

\section{Die Zusammensetzung des Peptons.}

Um die Einheitlichkeit des neuen Peptons zu prüfen, wurden nicht nur die Präparate sämtlicher Darstellungen (mit 
Ausnahme der ersten Darstellung, die nur zu Reaktionen Verwendung fand) analysiert, sondern auch wiederholt umgefällt und nach jeder Umfällung analysiert.

Die Trocknung der Substanz zur Ausführung der Elementaranalysen ging äußerst langsam von statten; ein Präparat war auch nach dem Trocknen im Trockenschrank bei $70^{\circ}$ innerhalb 6 Wochen noch nicht konstant, außerdem bewirkte die lange Trocknungsperiode eine teilweise Rückbildung des Peptons in Albumosen.

Trocknete ich bei $100^{\circ}$ aus, so wurde das Pepton nach 4-6 Tagen gelblich; der Stickstoffgehalt änderte sich wesentlich.

Zur Beschleunigung der Trocknung des Peptons konstruierte ich mir einen Exsiccator, der in der "Chemikerzeitung» 1903, Nr. 16, S. 27 näher beschrieben ist.

Die Elementaranalysen wurden in der üblichen Weise durch Verbrennung mit $\mathrm{CuO}$ und Bleichromat ausgeführt. Hierbei zeigte sich, daß die Substanz mit Ausnahme des Peptons der II. Darstellung völlig aschefei war. Der Stickstoffgehalt wurde teilweise nach Dumas, teilweise nach $\mathrm{Kjeldahl}$ bestimmt. Folgendes sind die gefundenen Werte für $\mathrm{C}, \mathrm{H}$ und $\mathrm{N}$ :

\section{Präparat der I. Verdauung}

wurde, zu kleiner Ausbeute wegen, nur zu Reaktionen verwendet.

Präparat der II. Verdauung

(Spuren Albumose enthaltend, Asche 0,38\%).

a) 0,2052 g Substanz gaben 0,3619 g CO $_{2}$ und 0,1259 g $\mathrm{H}_{2} \mathrm{O}$, d. i. $\mathrm{C}=48,09 \%$ und $\mathrm{H}=6,82 \%$;

b) 0,1407 . > $20,3 \mathrm{ccm}$ tr. $\mathrm{N}$ bei $19^{\circ}$ und $762 \mathrm{~mm}$ Bar., d. i. $\mathrm{N}=17,11 \%$.

Präparat der III. Verdauung

(völlig albumosefrei, fast S-frei, völlig aschefrei).

Nicht umgefällt.

a) 0,2236 g Substanz gaben $0,3965 \mathrm{~g} \mathrm{CO}_{2}$ und $0,1447 \mathrm{~g} \mathrm{H}_{2} \mathrm{O}$, d. i. $\mathrm{C}=48,35 \%$ und $\mathrm{H}=7,19 \%$;

b) 0,260 » $\gg 0,4612 \mathrm{~g} \mathrm{CO}_{2}$ und $0,1669 \mathrm{~g} \mathrm{H}_{2} \mathrm{O}$, d. i. $\mathrm{C}=48,38 \%$ und $\mathrm{H}=7,13 \%$;

c) $0,2097 \gg \quad$ \ $0,3728 \mathrm{~g} \mathrm{CO}_{2}$ und $0,1297 \mathrm{~g} \mathrm{H}_{2} \mathrm{O}$, d.i. $\mathrm{C}=48,48^{\circ} \%$ und $\mathrm{H}=6,87^{\circ} \%$; 
d) $0,2344 \mathrm{~g}$ Substanz gaben $35,0 \mathrm{ccm}$ tr. $\mathrm{N}$ bei $757,4 \mathrm{~mm}$ Bar. und $22,0^{\circ}$, d. i. $\mathrm{N}=17,22 \%$

e) 0,2348 . $\quad$ > $34,7 \mathrm{ccm}$ tr. $\mathrm{N}$ bei $20,2^{\circ}$ und $757,8 \mathrm{~mm}$ Bar., d. i. $\mathrm{N}=17,19 \%$.

Einmal umgefällt (ohne Essigsäure).

a) 0,2556 g Substanz gaben 0,4503 $\mathrm{g} \mathrm{CO}_{2}$ und 0,1619 $\mathrm{g} \mathrm{H}_{2} \mathrm{O}$, d.i. $\mathrm{C}=48,04 \%$ und $\mathrm{H}=7,03 \%$;

b) 0,2251 > $\gg 0,3952 \mathrm{~g} \mathrm{CO}_{2}$ und $0,1347 \mathrm{~g} \mathrm{H}_{2} \mathrm{O}$, d.i. $\mathrm{C}=47,84 \%$ und $\mathrm{H}=6,64 \%$;

c) $0,230 \gg, \quad, 0,4026 \mathrm{~g} \mathrm{CO}_{2}$ und $0,1408 \mathrm{~g} \mathrm{H}_{2} \mathrm{O}$, d.i. $\mathrm{C}=47,77 \%$ und $\mathrm{H}=6,81 \%$;

d) $0,1864, \quad, 26,7 \mathrm{ccm}$ tr. $\mathrm{N}$ bei $22^{\circ}$ und $763 \mathrm{~mm}$ Bar., d. i. $\mathrm{N}=16,64 \%$;

e) $0,2531 \gg \quad » 37,4 \mathrm{ccm}$ tr. $\mathrm{N}$ bei $20,5^{\circ}$ und $763 \mathrm{~mm}$ Bar., d. i. $\mathrm{N}=17,08 \%$.

Zweimal umgefällt.

a) 0,2622 g Substanz gaben $0,4623 \mathrm{~g} \mathrm{CO}_{2}$ und $0,1583 \mathrm{~g} \mathrm{H}_{2} \mathrm{O}$, d.i. $\mathrm{C}=48,09 \%$ und $\mathrm{H}=6,71 \%$;

b) 0,2321 » $>0,4062 \mathrm{~g} \mathrm{CO}_{2}$ und $0,1429 \mathrm{~g} \mathrm{H}_{2} \mathrm{O}$, d. i. $\mathrm{G}=47,74^{\circ} \%$ und $\mathrm{H}=6,84 \%$;

c) 0,1881 \ $\quad 0,3296 \mathrm{~g} \mathrm{CO}_{2}$ und $0,1187 \mathrm{~g} \mathrm{H}_{2} \mathrm{O}$, d. i. $\mathrm{C}=47,79 \%$ und $\mathrm{H}=7,01 \%$;

d) $0,2032 », \quad 28,3 \mathrm{ccm}$ tr. $\mathrm{N}$ bei $12^{\circ}$ und $764,5 \mathrm{~mm}$ Bar., d. i. $\mathrm{N}=16,78 \%$.

Dreimal umgefällt.

a) 0,2114 g Substanz gaben $0,3697 \mathrm{~g} \mathrm{CO}_{2}$ und $0,1270 \mathrm{~g} \mathrm{H}_{2} \mathrm{O}$, d.i. $\mathrm{C}=47,69 \%$ und $\mathrm{H}=6,67 \%$;

b) $0,1920>\quad \rightarrow 0,3375 \mathrm{~g} \mathrm{CO}_{2}$ und $0,1161 \mathrm{~g} \mathrm{H}_{8} \mathrm{O}$, d. i. $\mathrm{C}=47,94 \%$ und $\mathrm{H}=6,72 \%$

c) 0,2103 \# $\quad, 30,5 \mathrm{ccm}$ tr. $\mathrm{N}$ bei $11,5^{\circ}$ und $758 \mathrm{~mm}$ Bar., d. i. $\mathrm{N} 17,36 \%$.

\section{Präparat IV}

(völlig albumosefrei, fast S-frei, völlig aschefrei).

Nicht umgefällt.

a) $0,2232 \mathrm{~g}$ Substanz gaben $0,3955 \mathrm{~g} \mathrm{CO}_{2}$ und $0,1415 \mathrm{~g} \mathrm{H}_{2} \mathrm{O}$, d. i. $\mathrm{C}=48,32 \%$ und $\mathrm{H}=7,04 \%$;

b) $0,2686 », \quad 0,4738 \mathrm{~g} \mathrm{CO}_{2}$ und $0,1701 \mathrm{~g} \mathrm{H}_{2} \mathrm{O}$, d. i. $\mathrm{C}=48,02 \%$ und $\mathrm{H}=7,04 \%$;

c) 0,2005 » $, 0,3508 \mathrm{~g} \mathrm{CO}_{2}$ und $0,1209 \mathrm{~g} \mathrm{H}_{2} \mathrm{O}$, d. i. $\mathrm{C}=47,73 \%$ und $\mathrm{H}=6,70 \%$;

d) 0,2412 " » $9,4219 \mathrm{~g} \mathrm{CO}_{2}$ und $0,1476 \mathrm{~g} \mathrm{H}_{2} \mathrm{O}$, d. i. $\mathrm{C}=47,71 \%$ und $\mathrm{H}=6,79 \%$; 
e) $0,2566 \mathrm{~g} \mathrm{Substan} z$ gaben $37,6 \mathrm{ccm}$ tr. $\mathrm{N}$ bei $15^{\circ}$ und $784,5 \mathrm{~mm}$ Bar., d. i. $\mathrm{N}=17,11 \%$;

f) $0,2002 » \quad$ " $29,0 \mathrm{ccm}$ tr. $\mathrm{N}$ bei $14^{\circ}$ und $763 \mathrm{~mm}$ Bar., d. i. $\mathrm{N}=17,30 \%$.

Einmal umgefällt.

a) 0,2684 g Substanz gaben $0,4694 \mathrm{~g} \mathrm{CO}_{2}$ und $0,1608 \mathrm{~g} \mathrm{H}_{2} \mathrm{O}$, d. h. $\mathrm{C}=47,69 \%$ und $\mathrm{H}=6,66^{\circ} \%$;

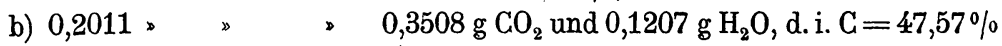
und $\mathrm{H}=6,66 \%$;

c) $0,1959 » \quad>\quad 28,2 \mathrm{ccm}$ tr. $\mathrm{N}$ bei $15^{\circ}$ und $7515,8 \mathrm{~mm}$ Bar., d. i. $\mathrm{N}=16,95^{\circ} \%$;

d) 0,2166 * $\quad 32,2 \mathrm{ccm}$ tr. $\mathrm{N}$ bei $20,2^{\circ}$ und $757,1 \mathrm{~mm}$ Bar., d. i. $\mathrm{N}=17,27 \%$.

Zweimal umgefällt.

a) 0,2505 g Substanz gaben 0,4392 $\mathrm{g} \mathrm{CO}_{2}$ und 0,1535 $\mathrm{g} \mathrm{H}_{2} \mathrm{O}$, d.i. $\mathrm{C}=47,82 \%$ und $\mathrm{H}=6,81 \%$;

b) $0,2053 \gg \quad$ » $\quad 0,3617 \mathrm{~g} \mathrm{CO}_{2}$ und $0,1245 \mathrm{~g} \mathrm{H}_{2} \mathrm{O}$, d. i. $\mathrm{G}=48,04 \%$ und $\mathrm{H}=6,74 \%$;

c) $0,1802 . » \quad \diamond 25,8 \mathrm{ccm}$ tr. $\mathrm{N}$ bei $12^{\circ}$ und $756 \mathrm{~mm}$ Bar., d. i. $\mathrm{N}=17,06 \%$.

Dreimal umgefällt.

a) 0,2717 g Substanz gaben 0,4755 $\mathrm{g} \mathrm{CO}_{2}$ und 0,1632 $\mathrm{g} \mathrm{H}_{\mathbf{2}} \mathrm{O}$, d.i. $\mathrm{C}=47,73 \%$ und $\mathrm{H}=6,67 \%$;

b) $0,2605 \triangleright \quad$ » $\quad 38,55 \mathrm{~cm} \mathrm{tr} . \mathrm{N}$ bei $19,5^{\circ}$ und $755,6 \mathrm{~mm}$ Bar., d. i. $\mathrm{N}=17,18 \%$;

c) 0,2086 \$ $\quad \gg 29,6 \mathrm{ccm}$ tr. $\mathrm{N}$ bei $15^{\circ}$ und $762,2 \mathrm{~mm}$ Bar., d. i. $\mathrm{N}=16,87 \%$.

Präparat der V. Verdauung (völlig albumosefrei, fast S-frei, völlig aschefrei).

Nicht umgefällt.

a) $0,2402 \mathrm{~g}$ Substanz gaben $0,4202 \mathrm{~g} \mathrm{CO}_{2}$ und $0,1483 \mathrm{~g} \mathrm{H}_{8} \mathrm{O}$, d. i. $\mathrm{C}=47,71 \%$ und $\mathrm{H}=6,86 \%$;

b) 0,1898 * * $\quad$, $0,3337 \mathrm{~g} \mathrm{COO}_{2}$ und $0,1816 \mathrm{~g} \mathrm{H}_{2} \mathrm{O}$, d. i. $\mathrm{C}=47,90^{\circ} \%$ und $\mathrm{H}=7,11 \%$ :

c) $0,2212 » \quad \gg 0,3863 \mathrm{~g} \mathrm{CO}_{2}$ und $0,1365 \mathrm{~g} \mathrm{H}_{2} \mathrm{O}$, d.i $\mathrm{C}=47,63 \%$ und $\mathrm{H}=6,85^{\circ} / 0$;

d) $0,1991 » \gg \quad 24,41 \mathrm{ccm} 1 / 10$-norm- $\mathrm{H}_{2} \mathrm{SO}_{4}$, d. i. $\mathrm{N}=17,16 \%$, Einmal umgefällt.

a) $0,1974 \mathrm{~g}$ Substanz gaben $0,3482 \mathrm{~g} \mathrm{CO}_{2}$ und $0,1187 \mathrm{~g} \mathrm{H}_{2} \mathrm{O}$, d.i. $\mathrm{C}=48,11 \%$ und $\mathrm{H}=6,68 \%$;

b) $0,2643 » \quad » 0,4636 \mathrm{~g} \mathrm{CO}_{2}$, d. i. $\mathrm{C}=47,83 \%$;

c) $0,1964 \gg \gg \quad 28,85 \mathrm{ccm}$ tr. $\mathrm{N}$ bei $16^{\circ}$ und $752,2 \mathrm{~mm}$ Bar. d. i. $\mathrm{N}=16,77 \%$. 
W. Scheermesser,

Zweimal umgefällt.

a) 0,2552 g Substanz gaben 0,4508 $\mathrm{g} \mathrm{CO}_{2}$ und 0,1541 $\mathrm{g} \mathrm{H}_{2} \mathrm{O}$, d. i. $\mathrm{C}=48,17 \%$ und $\mathrm{H}=6,70 \%$;

b) 0,2402 » $\gg 0,4214 \mathrm{~g} \mathrm{CO}_{2}$ und $0,1463 \mathrm{~g} \mathrm{H}_{2} \mathrm{O}$, d.i. $\mathrm{C}=47,84 \%$ und $\mathrm{H}=6,77 \%$;

c) $0,2086 » \quad \gg 29,1 \mathrm{ccm}$ tr. $\mathrm{N}$ bei $15^{\circ}$ und $756,3 \mathrm{~mm}$ Bar., d. i. $\mathrm{N}=16,45 \%$.

Präparat der VI. Verdauung.

Nicht umgefällt.

0,2198 g Substanz gaben 26,32 ccm 1/10-norm- $\mathrm{H}_{2} \mathrm{SO}_{4}$, d. i. $\mathrm{N}=16,76 \%$.

Gesamtübersicht der Analysen.

Präparat der II. Verdauung.

\begin{tabular}{|c|c|c|c|c|}
\hline & \multicolumn{3}{|c|}{ Nicht umgefällt } & Berechnet \\
\hline $\mathrm{C} \%$ & 48,09 & - & - & 48,1 \\
\hline $\mathrm{H} \%$ & 6,82 & - & - & 6,8 \\
\hline $\mathrm{N}^{0} / 0$ & 一 & 17,11 & 一 & 17,1 \\
\hline
\end{tabular}

Präparat der III. Verdauung.

\begin{tabular}{l|r|r|r|r|r|r|r|r|r|r|r|r|r|r}
\hline \hline & \multicolumn{2}{|c|}{$\begin{array}{c}\text { Nicht } \\
\text { umgefällt }\end{array}$} & \multicolumn{2}{c}{$\begin{array}{c}\text { Einmal } \\
\text { umgefällt }\end{array}$} & \multicolumn{2}{c|}{$\begin{array}{c}\text { Zweimal } \\
\text { umgefällt }\end{array}$} & $\begin{array}{c}\text { Dreimal } \\
\text { umgefällt }\end{array}$ & $\begin{array}{c}\text { Be- } \\
\text { rechnet }\end{array}$ \\
\hline $\mathrm{C} \%$ & 48,35 & 48,38 & 48,48 & 48,04 & 47,84 & 47,77 & 48,09 & 47,74 & 47,79 & 47,69 & 47,94 & 48,1 \\
$\mathrm{H} \%$ & 7,19 & 7,13 & 6,87 & 7,03 & 6,64 & 6,81 & 6,71 & 6,84 & 7,01 & 6,67 & 6,72 & 6,8 \\
$\mathrm{~N} \%$ & 17,22 & 17,19 & - & 16,64 & 17,08 & - & 16,78 & - & - & 17,36 & - & 17,1
\end{tabular}

Präparat der IV. Verdauung.

\begin{tabular}{|c|c|c|c|c|c|c|c|c|c|c|c|c|}
\hline & \multicolumn{3}{|c|}{$\begin{array}{c}\text { Nicht } \\
\text { umgefällt }\end{array}$} & \multicolumn{3}{|c|}{$\begin{array}{c}\text { Einmal } \\
\text { umgefällt }\end{array}$} & \multicolumn{3}{|c|}{$\begin{array}{c}\text { Zweimal } \\
\text { umgefällt }\end{array}$} & \multicolumn{2}{|c|}{$\begin{array}{c}\text { Dreimal } \\
\text { umgefällt }\end{array}$} & $\begin{array}{l}\mathrm{Be}- \\
\text { rechnet }\end{array}$ \\
\hline $\mathrm{C} \%$ & 48,32 & 48,02 & 47,73 & 47,69 & 47,50 & - & 47,82 & 48,04 & - & 47,73 & - & 48,1 \\
\hline $\mathrm{H} \%$ & 7,04 & 7,04 & 6,70 & 6,66 & 6,66 & - & 6,81 & 6,74 & - & 6,67 & - & 6,8 \\
\hline$N^{0} / 0$ & 17,11 & 17,30 & - & 16,95 & - & 17,27 & - & - & 17,06 & 17,18 & 16,87 & 17,1 \\
\hline
\end{tabular}


Präparat der V. Verdauung.

\begin{tabular}{|c|c|c|c|c|c|c|c|c|}
\hline \multirow[b]{2}{*}{$\mathrm{C} \%$} & \multicolumn{3}{|c|}{ Nicht umgefällt } & \multicolumn{2}{|c|}{$\begin{array}{c}\text { Einmal } \\
\text { umgefällt }\end{array}$} & \multicolumn{2}{|c|}{$\begin{array}{l}\text { Zweimal } \\
\text { umgefällt }\end{array}$} & \multirow{2}{*}{$\frac{\text { Berechnet }}{48,1}$} \\
\hline & 47,71 & 47,90 & 47,63 & 48,11 & 47,8 & \begin{tabular}{l|l|}
3 & 48,17
\end{tabular} & 47,84 & \\
\hline $\mathrm{H}^{0} / 0$ & 6,86 & 7,11 & 6,85 & 6,68 & - & 6,70 & 6,77 & 6,8 \\
\hline \multirow[t]{4}{*}{$\mathrm{N}^{0} / 0$} & 17,16 & - & - & 16,77 & - & 16,45 & - & 17,1 \\
\hline & \multicolumn{8}{|c|}{ Präparat der VI. Verdauung. } \\
\hline & & & \multicolumn{3}{|c|}{ Nicht umgefällt } & \multicolumn{2}{|c|}{ Berechnet } & \\
\hline & & $\mathrm{N}$ & & 16,76 & & 17,1 & & \\
\hline
\end{tabular}

Aus den gefundenen Zahlen berechnet sich als einfachste Formel für das Pepsin-Glutinpepton:

$$
\mathrm{C}_{23} \mathrm{H}_{39} \mathrm{~N}_{7} \mathbf{O}_{10} \text {. }
$$

\section{Zink- und Barytsalze des Pepsin-Glutinpeptons.}

Zur Darstellung der Zinksalze wurde chemisch reines Zinkoxyd von «Kahlbaum» mehrere Male mit Wasser ausgekocht und mit einer Lösung von Pepton bis zum Aufkochen erhitzt. Nach dem Erkalten wurde filtriert, nachgewaschen und die Lösung bis zur dünnen Sirupkonsistenz auf dem Wasserbade eingeengt. Diese Flüssigkeit wurde in absolutem Alkohol unter kräftigem Umrühren gefällt. Hierbei schied sich das Zinksalz in Form von Nadeln ab, die aber keine Kristalle waren. Der Niederschlag wurde abgesaugt, mit Alkohol und Äther nachgewaschen und sodann zunächst im Exsiccator, darauf im Trockenschrank bei $70^{\circ}$ getrocknet. Das Trocknen ging gut von statten : es bedurfte ca. $1 \mathrm{~g}$ Salz nur etwa 8 Tage, um konstant zu sein.

Bei der Darstellung der Barytsalze wurde das Pepton zunächst in einer frisch bereiteten Barythydratlösung gelöst und dann $\mathrm{CO}_{2}$ bis zur eben noch alkalischen Reaktion eingeleitet. Nach dem Aufkochen und Erkalten wurde filtriert. Das Filtrat wurde, wie beim Zinksalz, eingeengt und in Alkohol gefällt.

Folgendes sind die gefundenen $\mathrm{Zn}-, \mathrm{Ba}-$ und $\mathrm{N}-\mathrm{W}^{\top}$ erte der dargestellten Zink- bezw. Barytsalze der verschiedenen Verdauungen:

Hoppe-Seyler's Zeitschrift f. physiol. Chemie. XLI. 
Präparat der II. Verdauung.

a) 0,2772 g Zn-Salz gaben 0,0173 g ZnO, $\mathrm{Zn}=5,00 \%$;

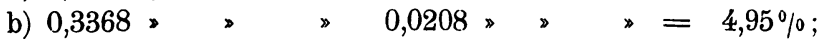

c) $0,4258 \gg \mathrm{Ba}-\mathrm{Salz} \gg 0,0724 \gg \mathrm{BaSO}_{4}, \mathrm{Ba}=10,0^{\circ} \%$.

Präparat der III. Verdauung.

Zinksalze.

Aus nicht umgefälltem Pepton.

a) $0,5767 \mathrm{~g} \mathrm{Zn-Salz} \mathrm{gaben} 0,0371 \mathrm{~g} \mathrm{Zn0,} \mathrm{Zn}=5,15 \%$;

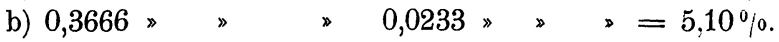

Aus einmal umgefälltem Pepton.

a) 0,4099 g Zn-Salz gaben 0,025̃5 g $\mathrm{ZnO}, \mathrm{Zn}=4,98 \%$.

Aus zweimal umgefälltem Pepton.

a) 0,489 g Zn-Salz gaben $0,0299 \mathrm{~g} \mathrm{Zn0,} \mathrm{Zn}=4,88 \%$;

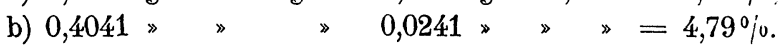

Barytsalze.

Aus nicht umgefälltem Pepton.

a) $0,2584 \mathrm{~g} \mathrm{Ba}-\mathrm{Salz}$ gaben $0,0440 \mathrm{~g} \mathrm{BaSO}_{4}, \mathrm{Ba}=10,0^{\circ}{ }_{i 0}$;

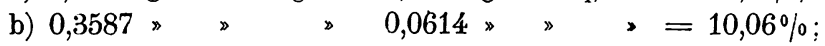

c) $0,200 \gg \quad \gg 26,3 \mathrm{ccm}$ tr. $\mathrm{N}$ bei $16^{\circ}$ und $765,1 \mathrm{~mm}$ Bar., $\mathrm{N}=15,63 \%$.

Präparat der IV. Verdauung.

Zinksalze.

Aus nicht umgefälltem Pepton.

a) $0,3085 \mathrm{~g} \mathrm{Zn-Salz} \mathrm{gaben} 0,0193 \mathrm{~g} \mathrm{ZnO}, \mathrm{Zn}=5,02 \%$;

b) $0,1818 \gg, \quad 24,7 \mathrm{ccm}$ tr. $\mathrm{N}$ bei $15^{\circ}$ und $764 \mathrm{~mm}$

Bar., $\mathrm{N}=16,19 \%$.

Aus einmal umgefälltem Pepton.

a) 0,3244 $\mathrm{g} \mathrm{Zn-Salz} \mathrm{gaben} 0,0198 \mathrm{~g} \mathrm{ZnO}, \mathrm{Zn}=4,90^{\circ} / 0$;

b) $0,2444 \gg \quad \gg 33,2 \mathrm{ccm}$ tr. $\mathrm{N}$ bei $10^{\circ}$ und $746 \mathrm{~mm}$ Bar., $\mathrm{N}=16,09 \%$.

Aus zweimal umgefälltem Pepton.

a) $0,3371 \mathrm{~g} \mathrm{Zn-Salz} \mathrm{gaben} 0,021 \mathrm{~g} \mathrm{ZnO}, \mathrm{Zn}=5,00^{\circ} \%$.

Aus dreimal umgefälltem Pepton.

a) 0,2620 g $\mathrm{Zn}-\mathrm{Salz}$ gaben $0,016 \mathrm{~g} \mathrm{ZnO}, \mathrm{Zn}=4,90 \%$;

b) $0,4011 » "$ » $0,0243 » \gg=4,85 \%$.

Barytsalze.

Aus nicht umgefälltem Pepton.

a) 0,4038 g Ba-Salz gaben $0,0721 \mathrm{BaSO}_{4}, \mathrm{Ba}=10,50 \%$;

b) 0,431 » $\gg 0,075 \gg>=10,23 \%$;

c) $0,2551 \gg \quad \gg 27,77 \mathrm{ccm}^{1 / 10}$-norm $-\mathrm{H}_{2} \mathrm{SO}_{4}, \mathrm{~N}=15,19 \%$. 


\section{Präparat der V. Verdauung. Zinksalze.}

Aus nicht umgefälltem Pepton.

a) 0,4492 g Zn-Salz gaben 0,0276 $\mathrm{g} \mathrm{Zn0,} \mathrm{Zn}=4,93 \%$.

Aus einmal umgefälltem Pepton.

a) 0,214 g Zn-Salz gaben $0,0137 \mathrm{~g} \mathrm{Zn0,} \mathrm{Zn}=5,13 \%$;

b) $0,2303 \gg$ » $26,82 \mathrm{ccm}{ }_{1}^{1 / 10}$-norm- $\mathrm{H}_{2} \mathrm{SO}_{4}, \mathrm{~N}=16,30 \%$. Barytsalze.

Aus zweimal umgefälltem Pepton.

a) $0,5398 \mathrm{~g} \mathrm{Ba-Salz} \mathrm{gaben} 0,0921 \mathrm{~g} \mathrm{BaSO}_{4}, \mathrm{Ba}=10,03 \%$;

b) $0,2012 \gg \quad \gg 22,28 \mathrm{ccm}^{1 / 10} /$ norm $^{-} \mathrm{H}_{2} \mathrm{SO}_{4}, \mathrm{~N}=15,50^{\circ} / 0$.

Zinksalze.

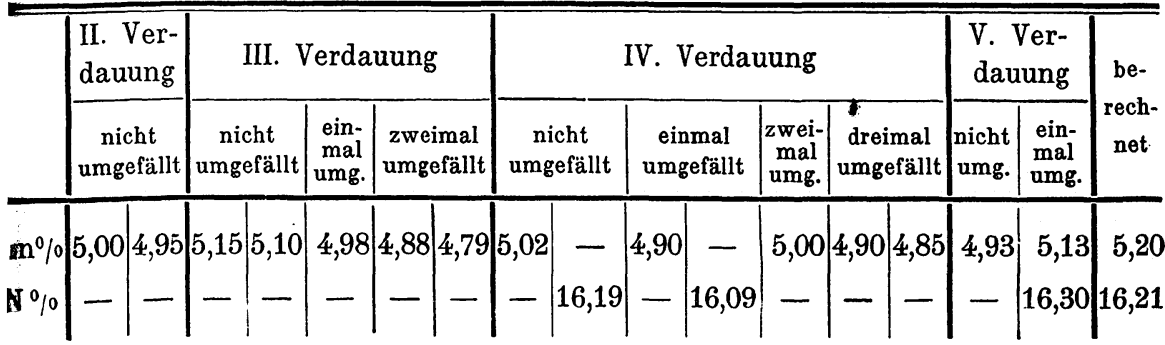

Barytsalze.

\begin{tabular}{|c|c|c|c|c|c|c|c|c|c|c|}
\hline & $\begin{array}{l}\text { II. Ver- } \\
\text { dauung }\end{array}$ & III. & Verdau & ung & IV. & Verdau & aung & V. Ver & lauung & be- \\
\hline & $\begin{array}{c}\text { nicht } \\
\text { umgefällt }\end{array}$ & nich & t umge & & nich & $t$ umge & fälltt & $\begin{array}{l}\mathbf{z w} \epsilon \\
\text { umg }\end{array}$ & $\begin{array}{l}\text { mal } \\
\text { fällt }\end{array}$ & net \\
\hline $\mathrm{Ba} \%$ & 10,00 & 10,00 & 10,06 & - & 10,50 & 10,23 & - & 10,03 & - & 10,69 \\
\hline $\mathrm{N} \%$ & - & - & - & 15,63 & - & - & 15,19 & - & 15,50 & 15,30 \\
\hline
\end{tabular}

\section{Drehung der Polarisationsebene durch das Pepsin-Glutinpepton.}

Wie alle bisher bekannten Peptone optisch aktiv, und zwar linksdrehend, sind, so kommt auch dem Leimpepton diese Eigenschaft zu. Zur Bestimmung der spezifischen Drehung des Präparates trocknete ich ca. $0,2 \mathrm{~g}$ Substanz bei $65^{\circ}$ nach Vortrocknung im Exsiccator bis zur Gewichtskonstanz und löste

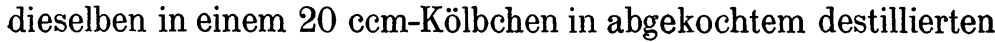


Wasser auf. Polarisiert wurde in einem Halbschattenapparat, der eine Einstellung auf $0,01^{\circ}$ gestattet. Bezeichne ich mit c den Prozentgehalt der Lösung, mit t Temperatur, L Rohrlänge in Dezimeter, $\alpha$ die Ablenkung, so sind folgende die gefundenen Werte, aus denen sich $\alpha[D]$ berechnet.

Präparat der III. Verdauung.

Nicht umgefällt.

$$
\begin{aligned}
& \text { 1. } \mathrm{c}=5,267 \\
& \mathrm{t}=20^{\circ} \\
& \text { daraus } \\
& \text { 2. } c=2,12 \\
& \mathrm{~L}=1 \\
& \alpha=-4,31^{\circ} \\
& \begin{array}{l}
\mathrm{t}=20^{\circ} \\
\mathrm{L}=2 \quad \text { daraus } \\
\alpha=-3,39^{\circ}
\end{array} \quad \alpha[\mathrm{D}]^{20}=-80,00^{\circ}
\end{aligned}
$$

Einmal umgefällt.

$$
\begin{aligned}
& \text { 1. } c=1,6: 32 \\
& \mathrm{t}=20^{\circ} \\
& \text { daraus } \\
& \text { 2. } \mathrm{c}=4,232 \\
& \mathrm{~L}=2 \\
& \alpha=-2,53^{\circ} \\
& \mathrm{t}=20,5^{\circ} \quad \text { daraus } \\
& { }^{\alpha}[\mathrm{D}]^{20}=-77,51^{\circ} \quad \mathrm{L}=2 \\
& \alpha[D]^{20,5}=-77,62^{\circ} \\
& \alpha=-6,57^{\circ} \\
& \mathrm{t}=20^{\circ} \\
& \mathrm{L}=4 \\
& \text { daraus } \alpha[D]^{20}=-77,9^{\circ} \\
& \alpha=-3,29^{\circ}
\end{aligned}
$$
1. $c=2,445$
$\mathrm{t}=20^{\circ}$
daraus
2. $\mathrm{c}=3,000$
$\mathrm{L}=4$
$\alpha=-7,65^{\circ}$$$
\mathrm{t}=20^{\circ}
$$$$
\text { daraus }
$$$$
\alpha[D]^{20}=-78,06^{\circ}
$$$$
\mathrm{L}=2
$$$$
\alpha=-4,67^{\circ}
$$

Präparat der IV. Verdauung.

Nicht umgefällt.
1. $c=1,110$
2. $c=2,70$
$\mathrm{t}=20^{\circ}$
daraus
$\mathrm{t}=20^{\circ}$
daraus
$\mathrm{L}=4$
$\alpha[D]^{20}=-76,13^{0}$
$\mathrm{L}=2$
$\alpha=-4,11^{\circ}$
$\alpha[D]^{20}=-76,11^{\circ}$
$\alpha=-3,38^{\circ}$

Ohne Essigsäure umgefällt.

$$
\begin{aligned}
& \mathrm{c}=2,196 \\
& \mathrm{t}=20^{\circ} \\
& \mathrm{L}=2 \quad \text { daraus } \quad \alpha[\mathrm{D}]^{20}=-84,01^{\circ} \\
& \alpha=-3,69^{\circ}
\end{aligned}
$$


Einmal umgefällt.
1. $\mathrm{c}=1,089$
$\mathrm{t}=18,5^{\circ}$
2. $\mathrm{c}=3,54$
$\mathrm{L}=2$
$\alpha=-1,68^{\circ}$
$\mathrm{t}=19^{\circ}$
daraus
$\mathrm{L}=2$
$\alpha[D]^{19}=-77,12^{\circ}$
$\alpha=-5,46^{\circ}$

Zweimal umgefällt.
1. $c=0,722$
2. $\mathrm{c}=1,982$
$\mathrm{t}=20^{\circ}$
$\mathrm{L}=2$
$\alpha=-1,12^{0}$
$\mathrm{t}=22^{\circ}$
daraus
$\mathrm{L}=2$
$\alpha[D]^{22}=-76,44^{\circ}$
daraus
$\alpha=-3,03^{\circ}$

Dreimal umgefällt.
1. $\mathrm{c}=1,231$
2. $c=3,741$
$\mathrm{t}=21,5^{0}$
daraus
$\mathrm{t}=18^{\circ}$
daraus
$\mathrm{L}=2$
$\alpha=-1,91^{\circ}$
${ }^{\alpha}[\mathrm{D}]^{21,50}=-77,58^{\circ}$
$\mathrm{L}=2$
$\alpha=-5,82^{\circ}$
${ }^{\alpha}[D]^{18}=-77,78^{\circ}$

Präparat der V. Verdauung.

Nicht umgefällt.

$$
\begin{aligned}
& \mathrm{c}=2,87 \\
& \mathrm{t}=20^{\circ} \quad \text { daraus } \alpha[\mathrm{D}]^{20}=-82,05^{\circ} \\
& \mathrm{L}=2 \\
& \alpha=-4,71^{\circ}
\end{aligned}
$$

Übersicht über die gefundenen Werte für $\alpha[D]$.

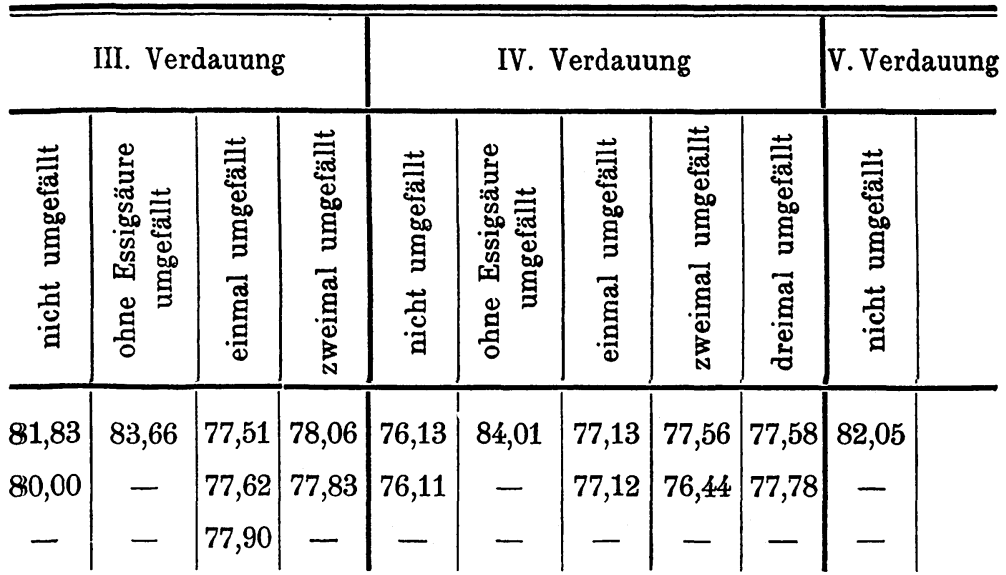

7 Molekulargewichtsbestimmung nach Beckmann.

Zur Molekulargewichtsbestimmung des Pepsinglutinpeptons durch Gefrierpunktserniedrigung wurde ausgekochtes Wasser als Lösungsmittel verwendet. 


$$
\begin{aligned}
& \left.\begin{array}{r}
3,772 \\
\text { Nullpunkt } \\
3,770 \\
3,771
\end{array}\right\} \text { angenommener Wert }=3,771^{\circ} . \\
& \text { Angewandte Menge Substanz }=0,3254 \mathrm{~g} . \\
& 3,666
\end{aligned}
$$

Erstarrungs-Punkt 3,665

3,661 angenommener Wert $=3,661$.

Depression $=0,110^{\circ}$.

Daraus ergibt sich nach der Formel $m=\frac{r \cdot p}{\Delta \cdot g}$ das Molekulargewicht $\mathrm{m}=558,4$.

Als Konstante für Wasser ist hierbei $1,89^{1}$ ) angenommen.

Aus der Formel $\mathrm{C}_{23} \mathrm{H}_{39} \mathrm{~N}_{7} \mathrm{O}_{10}$ berechnet sich das Molekulargewicht $\mathrm{zu} 573,2$.

Das gefundene Molekulargewicht stimmt scheinbar mit dem berechneten überein, wenigstens soweit, als man dies bei einem so hochmolekularen Körper erwarten kann; trotzdem dürfte das gefundene Molekulargewicht $\mathrm{zu}$ niedrig sein, wenn man die Zerfallsprodukte des Leimpeptons in Betracht zieht; jedenfalls ist das wirkliche Molekulargewicht ein mehrfaches der gefundenen Zahl, mindestens aber doppelt so groß.

\section{Spaltungsversuche.}

Ich führte im ganzen drei Spaltungsversuche aus: den ersten mit $14 \mathrm{~g}$ Substanz IV. Darstellung, die beiden andern mit je $6 \mathrm{~g}$ III. bezw. V. Darstellung. Bei der mit $14 \mathrm{~g}$ Pepton ausgeführten Spaltung kam es mir vorzugsweise darauf an, die entstandenen Produkte zu isolieren, bei den beiden andern Spaltungen hauptsächlich die Verteilung des Stickstoffs im Peptonmolekül in quantitativer Beziehung, als Amid-, Monoamino- und Diamino-N, festzustellen. Aber auch bei der ersten Spaltung aus $14 \mathrm{~g}$ Pepsin-Glutinpepton, bei welcher die erhaltenen Spaltungsprodukte isoliert wurden, wurde gleichzeitig die Verteilung des Stickstoffs festgestellt und bei der Gewinnung der erhaltenen Spaltungsprodukte, soweit dies möglich war, quantitativ gearbeitet.

1) E. Beckmann, Über die Methode der Molekulargewichtsbestimmung durch Gefrierpunktserniedrigung. Ztschr. f. Physik. Chemie, Bd. II, 1888, S. 716. 
Erster Spaltungsversuch.

Spaltung von $14 \mathrm{~g}$ Pepsin-Glutinpepton, IV. Verdauung.

$14 \mathrm{~g}$ Pepton wurden mit einer Mischung von $100 \mathrm{~g}$ Wasser und $50 \mathrm{~g}$ konzentrierter Schwefelsäure 12 Stunden lang am Rückflußkühler gekocht, mit Wasser verdünnt und auf $500 \mathrm{ccm}$ aufgefüllt. Die Lösung war somit 2,8\% ig.

Hiervon wurden je zweimal $5 \mathrm{ccm}$ (entsprechend ca. 0,14 g Pepton) kjeldahlisiert.

a) $5 \mathrm{ccm}$ brauchten $16,94 \mathrm{ccm}{ }^{1 / 10}$-norm- $\mathrm{H}_{2} \mathrm{SO}_{4}$.

b) $5 \mathrm{ccm}$ brauchten $16,98 \mathrm{ccm}{ }^{1 / 10}$-norm- $\mathrm{H}_{2} \mathrm{SO}_{4}$.

Im Mittel brauchten $5 \mathrm{ccm}=16,96 \mathrm{ccm}^{1} / 10-$ norm- $\mathrm{H}_{2} \mathrm{SO}_{4}$ entsprechend $0,023744 \mathrm{~g} \mathrm{~N}$.

Somit sind in den $500 \mathrm{ccm}$ Spaltungsflüssigkeit $2,3744 \mathrm{~g} \mathrm{~N}$. Da das Pepsin-Glutinpepton $17 \%$ Stickstoff enthält, entsprechen diese 2,3744 g $\mathrm{N}=13,96 \mathrm{~g}$ Pepton.

Nach Feststellung des N-Gehaltes wurde die Flüssigkeit mit $950 \mathrm{ccm}$ Wasser verdünnt, so daß dieselbe ca. $5 \% \mathrm{H}_{2} \mathrm{SO}_{4}$ enthielt, auf $65^{\circ}$ angewärmt und mit Phosphorwolframsäurelösung gefällt. Hierzu waren ca. $50 \mathrm{~g}$ feste Phosphorwolframsäure nötig. $\mathrm{Zu}$ bemerken ist, daß die von Kahlbaum bezogene Säure in allen Fällen, wo sie zur Verwendung kam, vorher durch Ausschütteln mit Äther aus der wässerigen Lösung und Einfließenlassen des Äthers in heißes Wasser (Verdampfen des Äthers) gereinigt wurde.

Bei der Fällung der Basen bildet sich ein voluminöser, käsiger Niederschlag von grauweißer Farbe, der sich leicht absetzte und sich schnell absaugen lie6. Er wurde mit 5\% iger kalter Schwefelsäure ausgewaschen.

\section{Der Phosphorwolframsäureniederschlag}

wurde nach Vorschrift Siegfrieds durch Anwärmen, unter Zusatz von etwas Ammoniak, in Wasser gelöst, die Phosphorwolframsäure und Schwefelsäure durch Baryt, das Baryum durch Ammonkarbonat entfernt. 
Zur Bestimmung des im Phosphorwolframsäureniederschlag enthaltenen Stickstoffs war es nötig, einmal die Niederschläge von $\mathrm{BaSO}_{4}$ und $\mathrm{Ba}$-Phosphorwolframat so gut als irgend möglich auszuwaschen, sodann das Ammonkarbonat der Lösung durch Eindampfen auf dem Wasserbade völlig zu entfernen. Der zurückbleibende Sirup wurde in $250 \mathrm{ccm}$ gelöst.

Von dieser Lösung brauchten

a) $10 \mathrm{ccm}=18,56 \mathrm{ccm}^{1} / 10-$ norm $-\mathrm{H}_{2} \mathrm{SO}_{4}$.

b) $10 \mathrm{ccm}=18,56 \mathrm{ccm}{ }^{1} / 10-$ norm $-\mathrm{H}_{2} \mathrm{SO}_{4}$.

Im Mittel brauchten

$10 \mathrm{ccm}=18,56 \mathrm{ccm}^{1} / 10$-norm- $\mathrm{H}_{2} \mathrm{SO}_{4}$, entsprechend $0,0260 \mathrm{~g} \mathrm{~N}$. Somit sind in den $250 \mathrm{ccm}$ Flüssigkeit $0,650 \mathrm{~g} \mathrm{~N}$, d. i. der Stickstoff des Phosphorwolframsäureniederschlages. Durch vorsichtigen Zusatz von Silbernitratlösung entstand in dieser Flüssigkeit ein flockiger Niederschlag. Die Abscheidung des Arginins geschah nach Kossels Vorschrift.

Der schnell braunschwarz werdende Niederschlag wurde, vor Licht geschützt, von der Flüssigkeit abfiltriert und mit Barytwasser nachgewaschen, sodann zur Entfernung des Ba und $\mathrm{Ag}$ mit $\mathrm{H}_{2} \mathrm{SO}_{4}$ und $\mathrm{HCl}$ geschüttelt und filtriert.

In dieser Lösung wurde nun wiederum ein Phosphorwolframsäureniederschlag erzeugt, der, wie vorher, von der Phosphorwolframsäure und Schwefelsäure befreit wurde. Die Lösung eingedampft, gab einen Syrup, dessen Gewicht $1,5 \mathrm{~g}$ betrug.

Nach Verdünnen des Sirups wurde nach der von Hedin ${ }^{1}$ ) angegebenen Methode auf "Histidin» geprüft. Diese Methode beruht auf der Fällbarkeit des Histidins durch Ammoniak und Silbernitratlösung.

Histidin war nicht vorhanden.

Bemerkt sei, daß auch Siegfried ${ }^{2}$ ) Histidin in seiner Arbeit bei der Zersetzung des «Glutokyrins» nicht nachweisen konnte.

1) Diese Zeitschrift, Bd. XXII, S. 192.

2) M. Siegfried, Zur Kenntnis der Hydrolyse des Eiweißes, Abdruck aus dem Bericht der math.-physik. Klasse der Königl. Sächs. Gesellschaft der Wissenschaften zu Leipzig vom 2. März 1903. 
Die mit $\mathrm{HNO}_{3}$ wieder schwach sauer gemachte Flüssigkeit wurde eingeengt, filtriert und mit Alkohol versetzt. Nach und nach wurde dieser Lösung Äther bis zur beginnenden Trübung zugegeben. Schon nach einem Tage hatten sich am Boden des Gefäßes büschelförmig gruppierte Nadeln abgesetzt. Als eine Vermehrung der Kristalle nicht mehr eintrat, wurden diese von der Mutterlauge getrennt und mit Alkohol, dann Äther nachgewaschen. Nach dem Trocknen über $\mathrm{H}_{2} \mathrm{SO}_{4}$ wurde der AgGehalt bestimmt.

$$
\begin{array}{cc}
0,1422 \mathrm{~g} \mathrm{Ag}-\mathrm{Salz} \text { gaben } 0,0392 \mathrm{~g} \mathrm{Ag} . \\
\mathrm{Ag} \text { gefunden } & \mathrm{Ag} \text { berechne } \\
27,50 \% & 26,55 \% .
\end{array}
$$

Da der Silbergehalt, auf Argininsilbernitrat berechnet, zu hoch war, wurde das Salz aus verdünntem Alkohol umkristallisiert. Danach ergaben:

$$
\begin{array}{cc}
0,2378 \mathrm{~g} \mathrm{Ag}-\mathrm{Salz} & =0,0630 \mathrm{~g} \mathrm{Ag} . \\
\mathrm{Ag} \text { gefunden } & \mathrm{Ag} \text { berechnet } \\
26,49 \% & 26,55 \%
\end{array}
$$

\section{N-Bestimmung:}

$0,1528 \mathrm{~g}$ Substanz gaben $27,3 \mathrm{ccm}$ tr. $\mathrm{N}$ bei $19^{\circ}$ und $750,2 \mathrm{~mm}$ Bar.

Gefunden:

$20,61 \% \mathrm{~N}$.
Berechnet für $\mathrm{C}_{6} \mathrm{H}_{14} \mathrm{~N}_{4} \mathrm{O}_{2} \cdot \mathrm{HNO}_{3} \cdot \mathrm{NO}_{3} \mathrm{Ag}$ :

$20,68 \%$ N.

Bestimmung der optischen Drehung für Argininsilbernitrat:

$$
\begin{aligned}
& \mathrm{c}=1,339 \\
& \mathrm{t}=20^{\circ} \\
& \mathrm{L}=2 \quad \text { daraus }{ }^{\alpha}[\mathrm{D}]^{20}=+6,30^{\circ} \\
& \alpha=+0,17^{\circ}
\end{aligned}
$$

Herr Professor Dr. Siegfried hatte die Güte, diese Polarisation selbst mit auszuführen. Gulewits $\mathrm{ch}^{1}$ ) fand für Argininsilbernitrat $a[D]^{20}=+5,60^{\circ}$.

Ich fand den Schmelz- und Zersetzungspunkt bei $184^{\circ}$, während Gulewitsch 180 bis $183^{\circ}$ angibt.

Das Filtrat vom Silberbarytniederschlag wurde nochmals filtriert und mit $\mathrm{HCl}$ und $\mathrm{H}_{2} \mathrm{SO}_{4}$ von $\mathrm{Ag}$ und $\mathrm{Ba}$ befreit; es wurde dann wiederum mit Phosphorwolframsäurelösung versetzt. Der entstandene Niederschlag wurde, wie vorher beschrieben, zersetzt, die Niederschläge sehr exakt ausgewaschen und die

1) Gulewitsch, Diese Zeitschr., Bd. XXVII. 
Flüssigkeiten zur Sirupkonsistenz eingeengt. Dieser Sirup wog 1,5 g. Da in ihm die Base Lysin vermutet wurde, wurde er mit $7,5 \mathrm{~g}$ Platinchlorid, d. i. die fünffache Menge des Sirups, vermischt und in ein weithalsiges Glas mit Alkohol gespült. Nach M. Siegfried ist es nämlich nötig, um ein leichtes Kristallisieren des Platindoppelsalzes von Lysin zu erreichen, die fünffache Menge Platinchlorid zu verwenden.

Nach und nach gab ich zu der alkoholischen Flüssigkeit Äther und setzte dies so lange fort, bis auf Zusatz von Äther eine Trübung nicht mehr entstand. Schon nach 12 Stunden hatten sich am Grunde des Gefäßes reichliche Kristalle in Form eines feinen Pulvers abgeschieden, das unter schwacher Vergrößerung als goldgelbe Kristallnädelchen zu erkennen war. Sie wurden nach acht Tagen abgesaugt, mit wasserfreiem Äther nachgewaschen und im Vacuum über Schwefelsäure getrocknet. Beim Trocknen verlor das feine, äußerst hygroskopische Kristallmehl seinen Glanz und ging in ein schmutziggelbes Pulver über. Es wog 2,365 g. Zur Identifizierung wurde der Pt-, C-, H- und $\mathrm{N}$-Gehalt bestimmt. Über Schwefelsäure getrocknet, wurden folgende Werte gefunden:

I. $0,2798 \mathrm{~g}$ Substanz gaben $0,0918 \mathrm{~g}$ Pt.

II. $0,2443 \mathrm{~g}$ Substanz gaben $10,9 \mathrm{ccm}$ tr. $\mathrm{N}$ bei $20^{\circ}$ und $732 \mathrm{~mm}$ Bar. III. 0,1468 g Substanz gaben 0,0505 $\mathrm{H}_{2} \mathrm{O}$ und $0,0864 \mathrm{~g} \mathrm{CO}_{\mathbf{2}}$.

\begin{tabular}{l|r|r}
\hline \hline Gefunden & $\begin{array}{r}\text { Berechnet für } \\
2 \mathrm{C}_{6} \mathrm{H}_{14} \mathrm{~N}_{2} \mathrm{O}_{2} \cdot \mathrm{Pt} \mathrm{Cl}_{6} \mathrm{H}_{2} \\
+\mathrm{C}_{2} \mathrm{H}_{5}(\mathrm{OH})\end{array}$ \\
\hline $\mathrm{Pt} \%$ & 32,88 & 32,37 \\
$\mathrm{C} \%$ & 16,05 & 15,95 \\
$\mathrm{H} \%$ & 3,82 & 3,69 \\
$\mathrm{~N} \%$ & 5,06 & 4,67
\end{tabular}

\section{Das Filtrat vom Phosphorwolframsäureniederschlag}

wurde auf $1^{1 / 2}$ l aufgefüllt und davon zweimal in je $10 \mathrm{ccm}$ der Stickstoff bestimmt.

Die Zerstörung der organischen Substanz durch $\mathrm{H}_{2} \mathrm{SO}_{4}$ bei Gegenwart von Phosphorwolframsäure geht äußerst langsam vor sich; sodann aber auch findet häufiges explosives Stoßen 
der Kjeldahlkolben statt. ${ }^{1}$ ) Auch Hausmann erwähnt in seiner Arbeit diese Tatsachen. Er findet, daß man oft erst nach 20 Stunden eine vollständige Zersetzung der Substanz erreicht.

$10 \mathrm{ccm}$ der auf $1 \frac{1 / 2}{1}$ aufgefüllten Flüssigkeit brauchten

a) $=8,38 \mathrm{ccm} \quad 1 / 10$-norm- $\mathrm{H}_{2} \mathrm{SO}_{4}$.

b) $=8,05 \mathrm{ccm} 1 / 10-$ norm- $\mathrm{H}_{2} \mathrm{SO}_{4}$.

Im Mittel brauchten

$10 \mathrm{ccm}=8,21 \mathrm{ccm}^{1 / 10}$-norm- $\mathrm{H}_{2} \mathrm{SO}_{4}$ entsprechend $0,011494 \mathrm{~g} \mathrm{~N}$.

Nach Feststellung des Stickstoffgehaltes wurde aus der Flüssigkeit die Phosphorwolframsäure entfernt und dann auf ca. $100 \mathrm{ccm}$ eingeengt.

In dieser Lösung wurde durch allmähliches umschichtiges Zusetzen von ammoniakalischer Silbernitratlösung und Silbernitratlösung eine Fällung erzeugt. Der sich sehr rasch absetzende Niederschlag wurde abgesaugt, mit Wasser, Alkohol und schließlich Äther nachgewaschen und im Vacuum über Schwefelsäure getrocknet. Die Ausbeute betrug 4,7 g.

0,1615 g Substanz gaben $0,0957 \mathrm{~g} \mathrm{Ag}$. berechnet

gefunden für glutaminsaures $\mathrm{Ag}$

$59,29 \% \mathrm{Ag}$ $59,83 \% \mathrm{Ag}$.

Aus dem Silbersalz wurde nun Glutaminsäurechlorhydrat dargestellt. Dieses wurde im Neubauerschen Tiegel abgesaugt, mit kalter rauchender $\mathrm{HCl}$ nachgewaschen und im Vacuum über Natronkalk getrocknet.

I. $0,1496 \mathrm{~g}$ Substanz gaben $0,1148 \mathrm{~g} \mathrm{AgCl}=0,0284 \mathrm{~g} \mathrm{Cl}$.

II. $0,1698 \mathrm{~g}$ Substanz gaben $=9,55 \mathrm{ccm} \quad 1 / 10$-norm- $\mathrm{H}_{8} \mathrm{SO}_{4}$ entsprechend $0,01337 \mathrm{~g}$ N.

$\begin{array}{cc}\text { Gefunden } & \text { berechnet } \\ \text { Cl } 18,98 \% & 19,31 \% \\ \mathrm{~N} \quad 7,87 \% & 7,74 \%\end{array}$

Es lag somit Glutaminsäurechlorhydrat vor. Zur weiteren Charakterisierung dieses Körpers wurde das optische Drehungsvermögen bestimmt.

1) Der inzwischen von M. Siegfried (Diese Zeitschr., dieses Heft, S. 1) beschriebene Kjeldahlapparat stand mir noch nicht zur Verfügung. 
Das Präparat drehte die Polarisationsebene nach rechts. $0,6576 \mathrm{~g}$ wurden in $20 \mathrm{ccm} 20 \%$ iger $\mathrm{HCl}$ gelöst.

$$
\begin{aligned}
& c=3,288 \% \\
& \mathrm{t}=20,5^{\circ} \\
& \mathrm{L}=2 \\
& \alpha=+1,61^{\circ} .
\end{aligned}
$$

Daraus berechnet sich für

$$
\begin{gathered}
\mathrm{C}_{3} \mathrm{H}_{5} \mathrm{NH}_{2}(\mathrm{COOH})_{2} \cdot \mathrm{HCl} \\
\alpha[\mathrm{D}]^{20,5}=+24,48^{\circ},
\end{gathered}
$$

für freie Glutaminsäure

$$
\alpha[\mathrm{D}]^{20,5}=+30,56^{\circ} \text {. }
$$

Emil Fischer und Th. Doerpinghaus, ${ }^{1}$ ) die unter den gleichen Bedingungen (Lösen in 20\% iger $\mathrm{HCl}$ ) wie ich das spezifische Drehungsvermögen der Glutaminsäure bestimmten, fanden dasselbe für Glutaminsäure aus

$$
\begin{aligned}
& \text { Horn }=+31,91^{\circ} \\
& \text { Casein }=+28,21^{\circ} \\
& \text { Gelatine }=+30,85^{\circ}
\end{aligned}
$$

während Scheibler ${ }^{2}$ ) einen Wert von $+25,5^{\circ}$ angab.

In neuerer Zeit bestimmte außer Fis cher noch Schukow ${ }^{3}$ ) das spezifische Drehungsvermögen der Glutaminsäure, gewonnen aus Melasseschlempe der Dessauer Strontianraffinerie $z u+26,52^{\circ}$. Müller ${ }^{4}$ ) wiederum fand $\alpha[D]^{20}$ von Glutaminsäure aus "Antipepton $\alpha$ Siegfried» $\mathrm{zu}+30,62^{\circ}$.

Das Filtrat vom glutaminsauren Silber wurde mit Schwefelammonium entsilbert und dann auf dem Wasserbade eingedampft. Der hierbei verbleibende Rückstand wurde mit heißem Alkohol mehreremal extrahiert, der Rückstand in Wasser gelöst und in Alkohol von ca. $40^{\circ}$ Temperatur eingegossen. Zur vollständigen Lösung war dazu ca. 11 Alkohol nötig. Beim Erkalten schieden sich an den Seiten sowie am Boden des Becherdes Horns .

1) Diese Zeitschr., Bd. XXXVI, S. 475, zum Artikel: «Hydrolyse

2) Bericht der Deutschen chemischen Gesellschaft, Bd. 17, S. 1728.

3) Zeitschrift des Vereins der deutschen Zuckerindustrie, 1900, Bd. 50, S. 814 .

4) Dissertation: Beitrag zur Kenntnis des Antipeptons. Leipzig 1903. 
glases während drei Tagen gelblich gefärbte ca. $3 \mathrm{~mm}$ dicke, warzenförmige Gebilde aus, die dreimal umkristallisiert wurden. Getrocknet wogen sie $1,588 \mathrm{~g}$.

Zur Elementaranalyse wurde bei $70^{\circ}$ getrocknet.

I. $0,2024 \mathrm{~g}$ Substanz gaben $0,2404 \mathrm{CO}_{2}$ und $0,1233 \mathrm{H}_{2} \mathrm{O}$.

II. $0,1934 \mathrm{~g}$ Substanz gaben $25,83 \mathrm{~cm} 1 / 10$-norm- $\mathrm{H}_{2} \mathrm{SO}_{4}$ entsprechend $0,036162 \mathrm{~g} \mathrm{~N}$.

\begin{tabular}{c|r|r}
\hline \hline & gefunden & $\begin{array}{c}\text { berechnet für } \\
\mathrm{C}_{2} \mathrm{H}_{5} \mathrm{NO}_{2}\end{array}$ \\
\hline $\mathrm{C}$ & $32,39 \%$ & $31,96 \%$ \\
$\mathrm{H}$ & $6,76 \%$ & $6,71 \%$ \\
$\mathrm{~N}$ & $18,69 \%$ & $18,70 \%$
\end{tabular}

Der Schmelz-und Zersetzungspunkt wurde übereinstimmend in zwei Fällen gefunden: $234^{\circ}$.

In dem Filtrat vom glutaminsauren Silber war also Glykokoll vorhanden.

Es sind somit bei der Spaltung des Pepsin-Glutinpeptons als sicher isoliert worden:

$$
\begin{aligned}
& \text { Arginin, } \\
& \text { Lysin, } \\
& \text { Glutaminsäure, } \\
& \text { Glykokoll, }
\end{aligned}
$$

während mit Sicherheit die Abwesenheit von Histidin nachgewiesen werden konnte.

\section{Zweiter Spaltungsversuch.}

Spaltung von $6 \mathrm{~g}$ Pepsin-Glutinpepton III. Verdauung.

Bei diesem, wie auch im dritten Spaltungsversuch sollte die Verteilung des Stickstoffs im Peptonmolekül bestimmt werden. $\mathrm{Zu}$ diesem Zwecke wurden $6 \mathrm{~g}$ Pepton, III. Verdauung, mit einer Mischung von $40 \mathrm{~g}$ Wasser und $20 \mathrm{~g}$ konzentrierter Schwefelsäure 12 Stunden lang am Rückflußkühler gekocht, und diese Flüssigkeit, die einen Rückstand nicht enthielt und von goldgelber Farbe war, auf $250 \mathrm{ccm}$ aufgefüllt. 
Von dieser Flüssigkeit brauchten $5 \mathrm{ccm}=14,47 \mathrm{ccm}^{1} 110-$ norm $-\mathrm{H}_{2} \mathrm{SO}_{4}$ entsprechend $0,020244 \mathrm{~g} \mathrm{~N}$. Somit enthalten die $250 \mathrm{ccm}=1,0122 \mathrm{~g} \mathrm{~N}$, was, bei Annahme von 17\% $\mathrm{N}$ im Pepsin-Glutinpeptonmolekül, $5,954 \mathrm{~g}$ trockenem Pepton entspricht. In dieser Lösung wurde nun zunächst der

\section{Amidstickstoff}

bestimmt und dabei die von Müller (l. c.) zur Beobachtung empfohlenen Vorschriften eingehalten.

Insbesondere wurde das Magnesiumoxyd auf Ammoniak geprüft: es war völlig frei davon.

Von der Spaltungsflüssigkeit wurden $20 \mathrm{ccm}$ mit $20 \mathrm{~g} \mathrm{MgO}$ destilliert und das Destillat $+20 \mathrm{ccm}$ Vorlage $\left({ }^{1} / 10-\right.$ norm $\left.-\mathrm{H}_{2} \mathrm{SO}_{4}\right)$ zur Entfernung der $\mathrm{CO}_{2}$ (vom $\mathrm{MgO}$ ) gekocht.

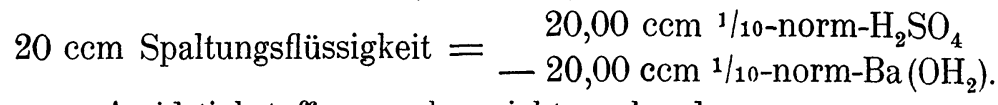
Amidstickstoff war also nicht vorhanden.

Bestimmung des Diaminostickstoffs

(Phosphorwolframsäureniederschlag).

Nach Entfernung der Phosphorwolframsäure und Schwefelsäure, wie dies im I. Spaltungsversuch beschrieben wurde und Auffüllen der eingedampften Flüssigkeit auf $250 \mathrm{ccm}$ brauchten

a) $25 \mathrm{ccm}=16,28 \mathrm{ccm} \quad 1 / 10-$ norm- $\mathrm{H}_{2} \mathrm{SO}_{4}$

b) $25 \%=15,98$ "1/10-norm- $\mathrm{H}_{2} \mathrm{SO}_{4}$.

Im Mittel brauchten

$25 \mathrm{ccm}=16,13 \mathrm{ccm}$ Norm. ${ }^{-1} / 10-\mathrm{H}_{2} \mathrm{SO}_{3}$ entsprechend $0,022582 \mathrm{~g} \mathrm{~N}$.

Somit sind in dem Phosphorwolframsäureniederschlag $=0,22582 \mathrm{~g} \mathrm{~N}$. Dieser Stickstoff verteilt sich auf Arginin und Lysin.

Zur Ermittelung, wieviel $\mathrm{N}$ auf jede der beiden Basen kommt, wurde in dreimal $50 \mathrm{ccm}$ der Argininsilberbarytniederschlag erzeugt; derselbe wurde mit Barytwasser nachgewaschen und kjeldahlisiert.

Der Argininsilberbarytniederschlag

a) verbrauchte $20,54 \mathrm{ccm} 1 / 10-$ norm $-\mathrm{H}_{2} \mathrm{SO}_{4}$ 
b) Kolben gesprungen

c) verbrauchte $20,06 \mathrm{ccm} 1 / 10-$ norm $-\mathrm{H}_{2} \mathrm{SO}_{4}$.

Im Mittel brauchten

von $50 \mathrm{ccm}$ Niederschlag $=20,30 \mathrm{ccm} \cdot 1 / 10-$ norm- $\mathrm{H}_{2} \mathrm{SO}_{4}$ entsprechend $0,02842 \mathrm{~g} \mathrm{~N}$.

Somit kommen auf Arginin

$$
0,1421 \mathrm{~g} \mathrm{~N} \text {. }
$$

Das Filtrat vom Silberbarytniederschlag (Lysin).

Der Lysinstickstoff wurde im Filtrat bestimmt; in a) wurde das beim Zerstören der organischen Substanz durch $\mathrm{H}_{2} \mathrm{SO}_{4}$ sich bildende $\mathrm{BaSO}_{4}$ nicht entfernt. Hierdurch gestaltete sich das Erhitzen mit $\mathrm{H}_{2} \mathrm{SO}_{4}$ infolge Siedeverzuges zu einer schwierigen Operation. Für b) und c) wurde vor dem Erhitzen mit Schwefelsäure zu der Flüssigkeit $\mathrm{H}_{2} \mathrm{SO}_{4}$ im Überschuß zugegeben, aufgekocht und vom $\mathrm{BaSO}_{4}$ abfiltriert, das Baryumsulfat mit heißem Wasser ausgewaschen. Nach dem Eindampfen und Kjeldahlisieren brauchten
a) $=11,92 \mathrm{ccm} 3 / 10$-norm- $\mathrm{H}_{2} \mathrm{SO}_{4}$
b) $=11,31$ »1/10-norm- $\mathrm{H}_{2} \mathrm{SO}_{4}$
c) $=11,74 \quad$, 1/10-norm- $\mathrm{H}_{2} \mathrm{SO}_{4}$.

Im Mittel $=11,66 \mathrm{ccm}$ entsprechend 0,016324 $\mathrm{g} \mathrm{N}$.

Somit kommen auf Lysin

$$
0,08162 \mathrm{~g} \mathrm{~N} .^{1} \text { ) }
$$

Bestimmung des Monoaminostickstoffs im Filtrat

vom Phosphorwolframsäure niederschlag.

Das Filtrat vom Phosphorwolframsäureniederschlag betrug $255 \mathrm{ccm}$; der Niederschlag war mit einer Lösung von $5 \%$ Phosphorwolframsäure und 5\% Schwefelsäure nachgewaschen, wozu $85 \mathrm{ccm}$ nötig waren.

Filtrat und Waschflüssigkeit wurden vereinigt und auf $500 \mathrm{ccm}$ aufgefüllt. Hiervon brauchten

a) $25 \mathrm{ccm}=22,81 \mathrm{ccm} 1 / 10-$ norm- $\mathrm{H}_{2} \mathrm{SO}_{4}$;

b) $25 》=22,90$ "1/10-norm- $\mathrm{H}_{2} \mathrm{SO}_{4}$.

1) Die bei Gegenwart von Salpetersäure ausgeführten N-Bestimmungen geben etwas zu hohe Werte. M. Siegfried. 
Im Mittel brauchten

$25 \mathrm{ccm}=22,85 \mathrm{ccm}^{1 / 10}$-norm- $\mathrm{H}_{2} \mathrm{SO}_{4}$, entsprechend $0,03199 \mathrm{~g} \mathrm{~N}$.

Somit sind in diesen $500 \mathrm{ccm}$ Filtrat vom Phosphorwolframsäureniederschlag $0,6396 \mathrm{~g} \mathrm{~N}$.

Wieviel Monoaminostickstoff kommt hiervon auf Glutaminsäure?

Die Glutaminsäure wurde, wie im ersten Spaltungsversuch, mit ammoniakalischer Silbernitratlösung und $\mathrm{AgNO}_{3}$ gefällt. Ausbeute an über $\mathrm{H}_{2} \mathrm{SO}_{4}$ im Vacuum getrocknetem $\left(\mathrm{C}_{5} \mathrm{H}_{7} \mathrm{NO}_{4}\right) \mathrm{Ag}_{2}$ $=1,6112 \mathrm{~g}$. Da der Stickstoffgehalt des glutaminsauren Silbers sich auf $4,05 \%$ berechnet, so entspricht dies $0,06525 \mathrm{~g} \mathrm{~N}$.

Übersicht über die prozentische Verteilung des Stickstoffs im Pepsin-Glutinpeptonmolekül des II. Spaltungsversuches.

\begin{tabular}{|c|c|c|c|c|}
\hline Amid-N & \multicolumn{2}{|c|}{ Basen-N } & Monoamino-N & $\begin{array}{l}\text { Gesamt-N } \\
\text { statt } 100 \%\end{array}$ \\
\hline \multirow[t]{3}{*}{$0 \%$} & \multicolumn{2}{|c|}{$\begin{array}{c}24,79 \% \\
\text { Davon } \\
\text { kommen auf }\end{array}$} & $\begin{array}{c}70,21 \% \\
\text { Davon } \\
\text { kommen auf }\end{array}$ & $95,0 \%$ \\
\hline & Arginin & Lysin & Glutaminsäure & \\
\hline & $15,59 \%$ & $8,95 \%$ & $10,20 \%$ & \\
\hline
\end{tabular}

Dritter Spaltungsversuch.

Spaltung von $6 \mathrm{~g}$ Pepsin-Glutinpepton, V. Verdauung.

Der dritte Spaltungsversuch war die Parallele zum zweiten Spaltungsversuch. Die Ausführung war in allen Fällen genau die gleiche wie beim zweiten.

Die durch Spaltung von $6 \mathrm{~g}$ Pepton erhaltene Lösung wurde auf $250 \mathrm{ccm}$ aufgefüllt.

Von dieser Flüssigkeit brauchten

$5 \mathrm{ccm}=14,27 \mathrm{ccm}{ }^{1 / 10}$-norm- $\mathrm{H}_{2} \mathrm{SO}_{4}$, entsprechend $0,019978 \mathrm{~g} \mathrm{~N}$.

Somit enthalten die $250 \mathrm{ccm}=0,9989 \mathrm{~g} \mathrm{~N}$, was bei Annahme von $17 \% \mathrm{~N}$ im Pepsin-Glutinpeptonmolekül 5,876 g trockenem Pepton entspricht. 


\section{Amidstickstoff}

war nicht vorhanden:

$20 \mathrm{ccm}$ Spaltungsflüssigkeit $=\begin{aligned} & 20 \mathrm{ccm} \\ & 19,62\end{aligned} \stackrel{1 / 10-\text { norm- }-\mathrm{H}_{2} \mathrm{SO}_{4}}{1 / 10 \text {-norm- } \mathrm{Ba}(\mathrm{OH})_{2}}$.

Bestimmung des Basenstickstoffs

(Phosphorwolframsäureniederschlag).

$250 \mathrm{ccm}$ aufgefüllte Flüssigkeit.

Hiervon brauchten

a) $25 \mathrm{ccm}=16,47 \mathrm{ccm}{ }^{1 / 10}-$ norm- $\mathrm{H}_{2} \mathrm{SO}_{4}$

b) $25 \gg=16,08$ »1/10-norm- $\mathrm{H}_{2} \mathrm{SO}_{4}$.

Im Mittel brauchten

$25 \mathrm{ccm}=16,27 \mathrm{ccm}{ }^{1 / 10}$-norm- $\mathrm{H}_{2} \mathrm{SO}_{4}$, entsprechend $0,0277 \mathrm{~g} \mathrm{~N}$.

Somit sind in dem Phosphorwolframsäureniederschlag $0,277 \mathrm{~g} \mathrm{~N}$.

Weitere $50 \mathrm{ccm}$ wurden mit Silbernitrat gefällt und mit Barytpulver geschüttelt. Der Silberniederschlag

a) verbrauchte $18,92 \mathrm{ccm} 1 / 10$-norm- $\mathrm{H}_{2} \mathrm{SO}_{4}$

b) 》 19,43 »1/10-norm- $\mathrm{H}_{2} \mathrm{SO}_{4}$

c) Kolben gesprungen.

Im Mittel $=19,28 \mathrm{ccm} 1 / 10-$ norm $-\mathrm{H}_{2} \mathrm{SO}_{4}$, entprechend $0,026992 \mathrm{~g} \mathrm{~N}$.

Somit kommen auf Arginin 0,13496 g N.

Das Filtrat vom Silberbarytniederschlag (Lysin).
a) $=13,89 \mathrm{ccm}{ }^{1} / 10$-norm- $\mathrm{H}_{2} \mathrm{SO}_{4}$
b) $=12,77$ »1/10-norm- $\mathrm{H}_{2} \mathrm{SO}_{4}$
c) $=12.62 » 1 / 10-$ norm- $\mathrm{H}_{2} \mathrm{SO}_{4}$.

Die Bestimmung in a scheint fehlerhaft $\mathrm{zu}$ sein. Ich nehme deshalb das Mittel von $b$ und $c$.

Im Mittel $=12,69 \mathrm{ccm} 1 / 10-$ norm- $\mathrm{H}_{2} \mathrm{SO}_{4}$, entsprechend $0,01776 \mathrm{~g} \mathrm{~N}$.

Somit kommen auf Lysin 0,0888 $\mathrm{g} \mathrm{N}$.

Bestimmung des Monoaminostickstoffs im Filtrat vom Phosphorwolframsäureniederschlag.

Das Filtrat vom Phosphorwolframsäureniederschlag betrug

Hoppe-Seyler's Zeitschrift f. physiol. Chemie. XLI. 
$355 \mathrm{ccm}$, das 5\% Phosphorwolframsäure und 5\% Schwefelsäure enthaltende Waschwasser $105 \mathrm{ccm}$. Aufgefüllt auf $500 \mathrm{ccm}$.

Hiervon brauchten

a) $25 \mathrm{ccm}=$ Kolben gesprungen

b) $25 \gg=22,43 \mathrm{ccm} 1 / 10-$ norm- $\mathrm{H}_{2} \mathrm{SO}_{4}$

c) $25 »=23,09$ $\gg 1 / 10$-norm- $\mathrm{H}_{2} \mathrm{SO}_{4}$

Im Mittel brauchten

$25 \mathrm{ccm}=22,76 \mathrm{ccm}^{3 / 10}$-norm- $\mathrm{H}_{2} \mathrm{SO}_{4}$, entsprechend $0,0314 \mathrm{~g} \mathrm{~N}$.

Somit sind in den $500 \mathrm{ccm}$, also Monoaminostickstoff $=0,628 \mathrm{~g}$ enthalten.

Wieviel Monoaminostickstoff kommt hiervon auf Glutaminsäure?

Die Ausbeute des glutaminsauren Silbers, welches im Vacuum über Schwefelsäure getrocknet war, betrug 1,4702 g. Da der N-Gehalt des glutaminsauren Silbers sich auf $4,05 \%$ berechnet, so entspricht dies 0,05954 g N.

Übersicht über die prozentische Verteilung des $\mathrm{N}$ im Pepsin-

Glutinpeptonmolekül des III. Spaltungsversuchs.

\begin{tabular}{|c|c|c|c|c|}
\hline Amid-N & \multicolumn{2}{|c|}{ Basen-N } & Monoamino- $\mathrm{N}$ & $\begin{array}{r}\text { Gesamt-N } \\
\text { statt } 100 \%\end{array}$ \\
\hline \multirow[t]{3}{*}{$0 \%$} & \multicolumn{2}{|c|}{$\begin{array}{c}25,13 \% \\
\text { Davon } \\
\text { kommen auf }\end{array}$} & $\begin{array}{c}69,85 \% \\
\text { Davon } \\
\text { kommen auf }\end{array}$ & $94,98 \%$ \\
\hline & Arginin & Lysin & Glutaminsäure & \\
\hline & $14,89 \%$ & $9,80 \%$ & $11,15 \%$ & \\
\hline
\end{tabular}

Hierbei ist keine Korrektion für die Löslichkeit der Argininphosphorwolframates angebracht, durch welche die Werte für Basen-N und Arginin-N erhöht, die für Lysin- und Amidosäure-N erniedrigt wurden. 